\title{
Accesibilidad y políticas sociales en la Argentina reciente: una interpretación desde los saberes expertos en programas de transferencias monetarias
}

\author{
Martín Eduardo Hornes ${ }^{1}$; Carolina Gabriela Maglioni²
}

Recibido: 05 de noviembre de 2018; Revisada: 25 de diciembre de 2018; Aceptado: 22 de julio de 2019

Resumen. A través de un recorrido por la experiencia de institucionalización de los programas de transferencias monetarias en Argentina (2003-2015), este artículo propone una aproximación a la noción de accesibilidad en las políticas sociales desde la perspectiva de los saberes expertos y las instituciones estatales en la materia. Exploraremos la trayectoria de surgimiento y expansión de las transferencias monetarias argentinas para señalar la presencia de saberes expertos involucrados en la consolidación de las políticas sociales de entrega de dinero en efectivo a amplios sectores vulnerables de la sociedad. Dicho recorrido nos permitirá afirmar las transferencias monetarias como políticas que: transformaron los esquemas de la protección social y las formas de intervención estatal sobre los pobres y la pobreza; ampliaron la noción de accesibilidad de las políticas sociales, y contribuyeron a instalar nuevos debates políticos y jurídicos sobre las formas de concebir a los titulares de derechos de la asistencia estatal.

Palabras clave: políticas sociales; accesibilidad; saberes expertos; Argentina; transferencias monetarias.

Sumario: Introducción. 1. La experiencia argentina en las transferencias monetarias. 1.1 Condiciones de emergencia. 1.2 Accesibilidad como premisa de la expansión social monetaria (2003-2008). 1.3 La consolidación y la accesibilidad en las transferencias monetarias. 2. Las transferencias monetarias argentinas: intervención experta y formas plurales de accesibilidad. 3. Referencias bibliográficas.

Cómo citar: Hornes M. E.; Maglioni, C. G. (2020) Accesibilidad y políticas sociales en la Argentina reciente: una interpretación desde los saberes expertos en programas de transferencias monetarias, en Cuad. trab. soc. 33(1), 7787.

\section{Introducción}

En las últimas décadas proliferaron mundialmente una serie de políticas sociales centradas en transferencias de dinero dirigidas a los sectores pobres. Pese a las múltiples definiciones que han recibido, se trata de programas sociales de transferencias monetarias (TM) que guardan similitudes: suplantan la tradicional provisión de bienes y servicios por la entrega de dinero, y establecen condicionalidades en materia de salud y educación sobre los menores pertenecientes al hogar (controles sanitarios y asistencia escolar).
Desde sus inicios, las agencias de desarrollo y los expertos vinculados al Banco Mundial (BM) y al Banco Interamericano de Desarrollo (BID) participaron en instancias de evaluación y difusión de los programas de transferencias monetarias (Teichman, 2007). A partir de 2006, la Comisión Económica para América Latina (CEPAL) promovió la incorporación de estos programas como la principal estrategia de "combate contra la pobreza" en América Latina y de redefinición en las formas de protección social no contributiva. Dicha propuesta alcanzó su máximo esplendor con la consolidación de la noción de "Piso de Protección

\footnotetext{
Universidad de San Martín, Argentina. m_hornes@hotmail.com

2 Universidad de Buenos Aires, Argentina. carolinamaglioni@yahoo.com.ar
} 
Social", impulsada por la Organización de las Naciones Unidas (ONU).

La proliferación de las transferencias monetarias estuvo acompañada de una serie de debates entre saberes expertos de diferentes organismos nacionales, regionales e internacionales vinculados a las políticas sociales. Distintos trabajos (Lomelí, 2009; Sugiyama, 2011) reconstruyeron las experiencias consideradas emblemáticas en materia de transferencias monetarias - PROSPERA (México) y Bolsa Família (Brasil) - y dieron cuenta de múltiples estrategias globales de difusión movilizadas por actores expertos y diferentes organismos financieros internacionales.

En un trabajo reciente señalamos que las transferencias monetarias convirtieron las agendas de discusión de los organismos internacionales y regionales vinculados a la protección social e impulsaron las transformaciones de las políticas sociales monetizadas (Hornes y Maglioni, 2018). En este artículo nos valemos de dichas discusiones para proponer una perspectiva novedosa. Recorriendo la experiencia de monetización reciente de las políticas sociales en la Argentina, iniciaremos una reflexión sobre la accesibilidad considerando el plano de las decisiones políticas y técnicas que delimitan el horizonte de las necesidades sociales de los sectores pobres, al definir el enfoque, tipo, calidad y cobertura de las mismas ${ }^{3}$.

Entendemos a la accesibilidad como un concepto que se refiere a un vínculo complejo que se construye entre los sujetos y las instituciones que prestan un servicio socialmente reconocido (Stolkiner y Barcala, 2000; Carballeda, 2014; Elizalde y Maglioni, 2014) y, por lo tanto, entraña encuentros y desencuentros entre los actores involucrados.

Desde esta mirada, se plantea que en la accesibilidad a las políticas sociales se articulan dos actores que responden a lógicas particulares: la de los usuarios y la de los efectores. ${ }^{4}$ Por ende, este proceso de interacción supone, por su naturaleza, una relación asimétrica: la determinación principal está en quién ofrece "so- luciones", no en quién las necesita y demanda. Clemente (2010) sostiene que al definir una política se presupone que ya está definida la necesidad que la sustenta y que esta definición resulta plasmada en el tipo de respuesta que materializa la misma: la delimitación de las "necesidades" corresponde a los niveles decisorios en todos sus planos, pues son éstos quienes definen los satisfactores que se brindaran como "respuesta".

Teniendo en cuenta lo anterior, centraremos nuestra mirada sobre el rol de los saberes expertos ${ }^{5}$ en los procesos de legitimación y expansión de las transferencias monetarias en Argentina, considerando que constituye un ejemplo de importación de ideas referidas a los "programas emblemas" de la región. Sostenemos que Argentina representa un laboratorio monetario: las concepciones de los organismos internacionales, las experiencias regionales, los viajes de ideas entre expertos, las evaluaciones, y las reuniones y los encuentros sobre "buenas prácticas" forman parte del repertorio de actividades que tuvieron incidencia en las políticas de transferencias monetarias que conocemos en la actualidad.

Reconstruiremos la historia reciente de las transferencias monetarias argentinas recurriendo al análisis de documentos correspondientes a organismos internacionales -Banco Mundial (BM), Banco Interamericano de Desarrollo (BID), Organización de las Naciones Unidas (ONU) y Fondo Monetario Internacional (FMI)-, regionales - Comisión Económica para América Latina (CEPAL) y Organización de Estados Americanos (OEA)- y locales Agencia Nacional de la Seguridad Social (ANSES), Ministerio de Desarrollo Social (MDS), etc.-.

Nos interesa abordar el proceso de surgimiento de las transferencias monetarias para reconocer las particularidades del caso argentino, prestando atención a la intervención de las instituciones y los saberes expertos (funcionarios de organismos internacionales, regionales y estatales, especialistas en políticas sociales,

Sobre la perspectiva de accesibilidad que orienta el artículo, véase, Clemente, Molina Derteano y Roffler (2014).

Desde el lugar de los efectores, podemos identificar dos subsistemas: el de las decisiones políticas y técnicas que interviene en el diseño y formulación de las políticas y el de los efectores institucionales que actúan en la implementación de las mismas, cuya ingeniería cambia en función de las particularidades del sector (salud, educación, vivienda, etc.) (Clemente et al., 2014).

Los saberes expertos refieren no solo a los actores portadores de conocimientos sino también a campos de expertise: "formas de intervención en el campo del poder y de la producción de bienes materiales y simbólicos que remiten a un saber técnicamente fundado, ligado a una disciplina científica o a un campo profesional (...). La conformación de un dominio de expertise supone la construcción de legitimidad del discurso experto, de los instrumentos técnicos movilizados y de los expertos como portadores de esos discursos y esos saberes" (Vommaro y Morresi, 2012, pp. 13-14). 
expertos del tercer sector-ONG, fundaciones, etc.) en la formulación de las políticas ${ }^{6}$.

La organización de nuestro artículo guardará relación con los procesos de surgimiento de las transferencias monetarias argentinas y los contextos sociohistóricos locales particulares que garantizaron la ampliación de los programas de transferencias monetarias, incrementando, a su vez, las perspectivas sobre la accesibilidad en las políticas públicas. Para finalizar, reflexionaremos en torno a los desafíos que presenta la accesibilidad en la transformación reciente de las transferencias monetarias argentinas.

\section{La experiencia argentina en las transferencias monetarias}

\subsection{Condiciones de emergencia}

Tras la crisis económica, política, social e institucional que condujo al estallido social de la Argentina, en diciembre de 2001, el gobierno interino de Eduardo Duhalde (2002-2003) tomó algunas medidas económicas y sociales centrales para revertir los dramáticos indicadores que signaban el contexto7: 1) derogó la paridad cambiaria finalizando con la convertibilidad monetaria; 2) decretó la emergencia ocupacional, alimentaria y sanitaria para concentrar medidas de intervención social sobre los sectores más desfavorecidos; y 3 ) recibió a los representantes del "Frente Nacional contra la Pobreza" (FRENAPO), organización multisectorial impulsada por la Central de Trabajadores Argentinos (CTA) que, desde junio del 2001, promovía una estrategia de redistribución de ingresos a partir de tres componentes: un seguro de empleo y formación para los jefes/as de hogar desocupados, una asignación por hijo menor de 18 años y otra para los mayores de 60 años que no contaran con cobertura previsional.

Las medidas implementadas fueron consensuadas en lo que se conoció como el "Diálogo Social Argentino": una mesa de diálogo nacional impulsada por el Episcopado argentino y apoyada por el Programa de las Naciones
Unidas para el Desarrollo (PNUD), con representación de sectores políticos y organizaciones sociales. En este escenario, surgió el Plan Jefes y Jefas de Hogar Desocupados (PJJHD) "Derecho de inclusión social", en abril de 2002, y se constituyó como la primera experiencia masiva de transferencias monetarias en Argentina dirigida hacia los sectores postergados, sin acceso al empleo y en condiciones de extrema pobreza. Financiado por los recursos procedentes de retenciones a las exportaciones y, luego, ampliado por un prestamo del Banco Mundial, este Plan se instituyó como una transferencia monetaria destinada a jefes/as de hogar desocupado/as, que tuvieran, al menos, un hijo menor de 18 años a cargo o hijos con discapacidad sin límite de edad o cuyo cónyuge se encontrara en estado de gravidez, y no percibiesen ninguna otra prestación económica por parte del Estado.

El programa, bajo dependencia directa del Ministerio de Trabajo y Seguridad Social y con apoyo de la Administración Nacional de la Seguridad Social, establecía una transferencia mensual a condición de que los beneficiarios cumpliesen con algún tipo de contraprestación laboral establecida desde los entes municipales como órganos ejecutores de la política social. A su vez, instituía la obligatoriedad de la asistencia escolar de niños/as y adolescentes como así también el cumplimiento del plan de vacunación obligatorio.

Las características que adquirió el Plan Jefes y Jefas de Hogar Desocupados confirman los significados que empieza a adquirir el dinero transferido hacia los sectores pobres: Argentina comienza a inscribir las transferencias monetarias como intervenciones focalizadas en un contexto de crisis económica, con el objetivo de brindar una asistencia directa a las poblaciones en condición de pobreza extrema, garantizando estabilidad social y gobernabilidad.

En pos de analizar la construcción de accesibilidad a los programas de transferencias monetarias, retomaremos algunos aportes significativos referidos al proceso de diseño del Plan para observar el rol de los saberes expertos en la institucionalización de las transferen-

Para una ampliación de la temática en general y de la aproximación metodológica en particular, véase Hornes (2018) y Maglioni (2018).

Para julio del año 2002, según datos de la Encuesta Permanente de Hogares (EPH) del Instituto Nacional de Estadística y Censos (INDEC), Argentina alcanzaba un índice de desempleo del 21,5\%, el mayor registrado de su historia. La pobreza también marcaba una cifra hasta el momento desconocida alcanzando a más del $50 \%$ de la población (18.500.000 personas) de las cuales el $24,8 \%$ (8.700.000 personas) se encontraban en la línea de indigencia. 
cias monetarias como un nuevo paradigma de política social en la "lucha contra la pobreza" en Argentina.

El denominado "Derecho a la Inclusión Social" - como subtítulo del programa- respondió a la demanda impulsada por el propio presidente y los actores de la Mesa del "Diálogo Social" (Repetto Potenza y Vilas, 2005), y guarda una clara referencia con las reivindicaciones impulsadas por el Frente Nacional contra la Pobreza (FRENAPO).

Sin embargo, a pesar de la vinculación establecida entre "el derecho familiar a un umbral mínimo de dignidad y el deber de realizar una contraprestación", en el contexto de emergencia del Plan Jefes y Jefas de Hogar Desocupados cualquier tipo de contraprestación o condicionalidad sobre el dinero transferido quedaría de lado, en virtud de que "la primera prioridad era poner en marcha de manera urgente la transferencia monetaria para atemperar los niveles de pobreza y conflictividad social" (Uña, Lupica y Strazza, 2009, p. 22).

Una de las particularidades de Argentina en términos de transferencias monetarias reside en que, al igual que Brasil, posee antecedentes en los debates sobre la noción de "renta básica" (del inglés original basic income). En tal horizonte de discusiones se inscriben algunas de las propuestas que anteceden al Plan Jefes y Jefas de Hogar Desocupados, movilizadas por ciertos actores con expertise en políticas sociales pero que, en ningún momento, resultó convocado formalmente por el Estado para participar de las etapas de diseño del programa. Podemos mencionar:

1. la propuesta de "ingreso ciudadano" del Centro de Investigaciones en Políticas Públicas (CIEPP) en 1995. Esta obtuvo notoriedad en el debate público cuando, durante 1997, las entonces diputadas nacionales, Elisa Carca y Elisa Carrió, de la Unión Cívica Radical (UCR), presentaron un proyecto de ley que proponía la creación de un "ingreso ciudadano para la infancia" (INICI);

2. la iniciativa de "ingreso para el desarrollo humano" del año 1999, formulada por Irene Novacovsky y Claudia Sobrón, desde la
Asociación Argentina de Políticas Sociales (AAPS) con base en las experiencias mexicanas y brasileñas; $y$

3. la propuesta de "ingreso de desarrollo humano", también de 1999, desarrollada por Horacio Rodríguez Larreta y Gonzalo Robredo, ambos miembros del Grupo SOPHIA.

El análisis, en clave de accesibilidad de la emergencia de los programas de transferencias monetarias en Argentina, nos permite aproximarnos a la lectura de la situación social crítica del país por parte de los actores expertos y de los decisores políticos. Allí se observa el reconocimiento de la pobreza como un problema de causas exógenas a los sujetos: "la responsabilidad de la situación de pobreza se ubicó en el funcionamiento de la economía y las acciones paliativas, consideradas de 'urgencia', se adecuaron a satisfacer necesidades elementales al ritmo del crecimiento de la conflictividad social" (Arias, 2012, p. 181). De ahí que el objetivo de la transferencia monetaria fuera asegurar un nivel de consumo básico de las familias en situación de pobreza, a fin de aliviar el conflicto social. Por esta razón, las condicionalidades incluidas asumieron un rol secundario, siendo monitoreadas de manera laxa.

A continuación, profundizaremos nuestras reflexiones respecto de los saberes expertos y las prácticas de expertise en términos de transferencias monetarias en Argentina, incorporando referencias sobre las transformaciones del contexto político y social.

\subsection{Accesibilidad como premisa de la ex- pansión social monetaria (2003-2008)}

Con la asunción en 2003 como presidente de Néstor Kirchner ${ }^{8}$, se instalaron variables ideológicas y políticas específicas para sustentar las estrategias de intervención social, iniciándose un período de transformaciones que se sustentó en "cuatro pilares: las políticas laborales y de recomposición salarial, la creación de puestos de trabajo por la reactivación económica, la disminución del empleo no registrado y el aumento de la cobertura previsional así como de las medidas de transferencia de ingresos" (Kessler, 2014, p.71). Este último

Kirchner llegó a la presidencia después de haber alcanzado el segundo lugar en las elecciones presidenciales de 2003 con el $22,24 \%$ de los votos. Accedió a la presidencia luego de que Carlos Menem renunciara a presentarse a la segunda vuelta electoral, conocida como "balotaje". 
aspecto visibiliza la emergencia de un período de proliferación de políticas de transferencias monetarias en Argentina.

En un escenario de recuperación económica y de rehabilitación del mercado de trabajo, comenzó una reestructuración del Plan Jefes y Jefas de Hogar Desocupados, identificando dentro de los sectores asistidos a aquellos que podrían categorizarse como "inempleables" y "empleables". Esta categorización experta inscribe la emergencia de dos nuevas líneas de acción en términos de transferencias monetarias: el Programa Familias por la Inclusión Social (2005-2010), dependiente del Ministerio de Desarrollo Social (MDS), y el Seguro de Capacitación y Empleo (2006 hasta la actualidad), dependiente del Ministerio de Trabajo, Empleo y Seguridad Social.

El Programa Familias se creó en 2005 como resultado de una reformulación del Programa de Atención a Grupos Vulnerables - Subprograma de Ingreso para el Desarrollo Humano (PAGV-IDH), en funcionamiento desde 2002 con financiamiento del Banco Interamericano de Desarrollo. Dirigido a familias pobres con hijos menores de 18 años o a mujeres embarazadas que se encontrasen desocupadas y no percibieran ingresos económicos de ninguna otra índole (contributivos o no contributivos), se trataba de una transferencia monetaria mensual que definía a la madre como titular del subsidio, con montos de dinero que se calculaban estableciendo un importe mínimo para cada familia, y considerando adicionales mensuales, según mujer embarazada o por hijo (hasta un máximo de 5 hijos).

El Programa Familias establecía condicionalidades que tenían como principal responsable de su cumplimiento a las madres, en tanto que titulares del beneficio: asistencia escolar, vacunación y controles médicos de los hijos y de las embarazadas, y la participación en actividades de desarrollo personal, familiar y comunitario. Características que reflejarían la importación directa de las premisas que estructuraban los "programas emblema" en términos de transferencias monetarias: el programa Oportunidades de México (PROSPERA) y el Programa Bolsa Família de Brasil (Hornes y Maglioni, 2018).

Resulta interesante resaltar que, la emergencia de una intervención con estas cualida- des, implica la institucionalización de un programa que pone en movimiento un esquema donde las transferencias monetarias dirigidas a los sectores pobres en Argentina comienzan a inscribirse en el desarrollo de un sistema integral de protección social, en consonancia con lo que sucede en la región desde 2006 (CEPAL, 2006) $)^{9}$.

El Familias se convirtió en una de las bocas de salida del Plan Jefes y Jefas de Hogar Desocupados y, específicamente, como una estrategia dirigida a realizar un corte de género en la población oriunda del primer programa de transferencias monetarias: una selección focalizada en las mujeres vulnerables y/o jóvenes con hijos menores a cargo. Podríamos afirmar que en la categoría de "inempleables", utilizada para el caso argentino, se reinscriben las cualidades innatas que se le suelen atribuir a las mujeres bajo los programas: las transferencias monetarias que conciben como titular del beneficio a la madre o mujer responsable del hogar suponen una mejor utilización sobre el uso del dinero y una garantía de acumulación sobre el capital humano de los menores pertenecientes al hogar (Banco Mundial, 2009).

Durante el año 2006, surgió el Seguro de Capacitación y Empleo (SCyE) como una línea de política de capacitación e inserción laboral destinada a aquella porción de beneficiarios "empleables" que aún pertenecían al Plan Jefes y Jefas de Hogar Desocupados. De una extensión establecida en 24 meses de duración, este seguro implicaba la renuncia definitiva a este Plan y la asistencia - en calidad de condicionalidad - a las prestaciones típicas de los programas de empleo (capacitaciones, finalización escolar, prácticas laborales y bolsas de trabajo).

La cantidad de beneficiarios que conformaron el Seguro de Capacitación y Empleo fue notablemente menor, en comparación con la migración hacia el programa Familias. Esto se explica por dos cuestiones centrales, que movilizaron la emergencia de estas transferencias monetarias: 1) la mayor cantidad de titulares del Plan Jefes y Jefas de Hogar Desocupados estaba compuesta por mujeres desocupadas con menores a cargo y, por ende, incluidas en la categoría de "inempleables" y, 2) porque el Seguro buscaba ser un programa transitorio que promoviera la inserción de la mayor can-

\footnotetext{
Para una ampliación de la lectura del escenario político regional particular que da lugar a tales transformaciones, veáse Hornes y Maglioni (2018).
} 
tidad de beneficiarios en el mercado formal de trabajo.

No podemos dejar de prestarle atención a otro hecho significativo del período histórico que estamos reseñando. Desde 2003, se produjo un crecimiento notorio de las entregas de dinero por parte del Estado bajo el formato de pensiones sociales no contributivas (PNC). Las pensiones sociales no contributivas comenzaron a incrementarse debido a la incorporación de requisitos para la asignación de los recursos, en oposición a los criterios de cupos, establecidos desde su creación en 1948. Así, las transferencias monetarias dirigidas a madres de 7 hijos o más, personas inválidas y/o adultos mayores "pasaron de poco menos de 340 mil en 2003 a 1.543 .781 en 2014" (Lombardía y Rodríguez, 2015, pp. 13-14).

Observar la proliferación de programas de transferencias monetarias en Argentina nos resulta útil para señalar una multiplicidad de nuevas figuras sobre las cuales se comienzan a concentrar las entregas de dinero. Es decir, y tal y como venimos desarrollando desde nuestra perspectiva sobre la accesibilidad de las políticas sociales, los saberes expertos multiplicaron las transferencias monetarias sobre la creación de "nuevos titulares" de la asistencia monetaria estatal.

Mientras que el Plan Jefes y Jefas de Hogar Desocupados focalizaba la entrega de dinero en el jefe/a de hogar desocupado/a, estas nuevas líneas de intervención, impulsadas en un contexto de recuperación económica, apelaron a la configuración de "nuevos titulares". Podemos aseverar que las transferencias monetarias comienzan a inscribirse en las distintas figuras de la asistencia -mujeres, madres embarazas, menores de edad, desocupados, discapacitados, ancianos y hogares pobres y/o indigentes- y con nuevos atributos que exceden a la contención social -red de protección, consumo, rehabilitación (formas de desarrollo para los pobres)- y reincorporación al mercado de trabajo.

Asimismo, la proliferación de programas de transferencias monetarias durante este período implicó una expansión de la cobertura.
En este sentido, consideramos que el formato simplificado que subyace en la transferencia monetaria, como modalidad de intervención para resolver distintas problemáticas asociadas a la pobreza, amplía las posibilidades de masificación de esta prestación al flexibilizar las barreras que atraviesan a la accesibilidad y que obstaculizan el acceso de las personas a los diversos sistemas de acción social.

En el próximo apartado mostraremos un nuevo giro en torno a la accesibilidad donde el eje de la reinterpretación de los saberes expertos y las prácticas de expertise comienza a constituirse sobre la base de transferencias monetarias como formas de restauración de derechos a distintos grupos etarios y sociales.

\subsection{La consolidación y la accesibilidad en las transferencias monetarias}

Hacia 2008 el contexto económico internacional comenzó a desatar una crisis del capitalismo "financiarizado", cuyos efectos no demoraron en hacerse sentir en Argentina. Mientras que desde 2003 el país presentaba una tasa de crecimiento promedio anual del 7,6\%, durante el período 2008/2009 dicho indicador solo alcanzó el 0,9\% con impacto en materia de empleo y pobreza.

A este escenario internacional complejo se sumarían acontecimientos locales, tales como:

El recordado lock out o paro patronal del campo $^{10}$, en torno a la Resolución $\mathrm{N}^{\mathrm{o}} 125 / 2008$ del Ministerio de Economía, tendiente a establecer un sistema de retenciones impositivas móviles a la soja, el trigo y el maíz.

Las declaraciones del Papa Benedicto XVI en las que sostenía que las situaciones de exclusión y pobreza resultaban un "escándalo social" para Argentina, de las cuales se harían eco el arzobispo de Buenos Aires y presidente del Episcopado, cardenal Jorge Bergoglio ${ }^{11}$. Ciertos estudios académicos difundidos por los medios de prensa mostraban que la pobreza alcanzaba al $39 \%$ de la población ${ }^{12}$.

La derrota electoral del gobierno oficialista en las elecciones legislativas de 2009, sufrien-

10 Durante 129 días, las cuatro organizaciones más importantes del sector agroganadero argentino (Sociedad Rural Argentina, Confederaciones Rurales Argentina, CONINAGRO y Federación Agraria Argentina) impulsaron distintos cortes de ruta en todo el país y el desabastecimiento de mercaderías en las principales ciudades capitales.

11 Sobre las declaraciones del Papa Benedicto XVI y las repercusiones en el contexto local, véase: http://www.lanacion.com. ar/1159565-para-el-papa-la-pobreza-en-la-argentina-es-un-escandalo http://www.lanacion.com.ar/1159600-bergoglio-reitero-laadvertencia-del-papa-la-pobreza-es-escandalosa

12 Las cifras señaladas refieren al estudio realizado por el Barómetro de la Deuda Social, perteneciente a la Universidad Católica Argentina (UCA). 
do un fuerte retroceso en las cámaras de diputados y senadores.

Tal coyuntura generó un escenario de disputa propicio para que el oficialismo intentara revertir el mapa político, desplegando medidas de gran impacto social. Durante el segundo semestre de 2009, surgieron nuevas líneas de intervención en términos de transferencias monetarias dirigidas a distintos actores sociales como titulares de derechos (Abramovich y Pautassi, 2009). El dinero estatal volvió a recuperar un espacio central en la escena política con el horizonte de contener las demandas sociales y estabilizar las controversias, ampliar el espectro de la protección a nuevos grupos sociales, dinamizar el consumo, y promover el bienestar y el desarrollo.

En dicho escenario, la creación del "Programa Ingreso Social con Trabajo - Argentina Trabaja" implicó la incorporación de 100.000 personas, pertenecientes a los conglomerados urbanos de mayor relegación y vulnerabilidad social del conurbano bonaerense, a cooperativas de trabajo destinadas a la realización de obras públicas. En su discurso, la expresidenta, Cristina Kirchner, resaltó dos dimensiones centrales de los programas de transferencias monetarias, al incorporar el concepto de "ingreso social con trabajo": lo que "significa un abordaje diferente a los planes que normalmente con asistencialismo se han desarrollado en nuestro país" y al señalar que el problema de la Argentina no es la pobreza sino que "es la inequidad social y la distribución del ingreso" 13 .

Los principales ejes de intervención del programa se basaban en la inclusión social a través del trabajo, la capacitación con una perspectiva integral, y la promoción de la organización cooperativa, y sus beneficiarios, como aquellas personas pertenecientes a hogares en situación de alta vulnerabilidad social que no contasen con otro ingreso proveniente de: trabajo registrado, pensión, jubilación, programa social nacional o provincial incompatible.

En su esquema fundacional, el programa implicaba una actividad laboral concreta en una sede estatal municipal como condición para poder ser beneficiario de la transferencia monetaria, que se depositaba en una cuenta individual y personal en calidad de "monotributista" social. En la actualidad, el programa recibe la denominación de "Hacemos Futuro" e implica aspectos de finalización ("terminalidad") de todos los niveles educativos y espacios de formación profesional.

Sin embargo, la política de mayor envergadura e impacto en Argentina en términos de transferencias monetarias es la denominada "Asignación Universal Por Hijo para la Protección Social" (AUH), creada por decreto presidencial durante el mes de octubre de 2009. Comenzó a implementarse durante el primer trimestre de 2010 con el objetivo primordial de "equiparar el ingreso de aquellos niños cuyos padres no estuvieran incorporados al mercado de trabajo formal" y, por ende, "no recibieran la asignación por hijo estipulada en el régimen contributivo de asignaciones familiares" (MECON, 2009). La Asignación Universal Por Hijo para la Protección Social, en consonancia con otras transferencias monetarias de la región, posee la cualidad de centrarse en transferencias directas de dinero, específicamente fundamentadas y focalizadas sobre los menores pertenecientes al hogar y transferibles a los mayores responsables.

A mediados de abril del 2011 se creó la “Asignación Universal por Embarazo" (AUE) con el objetivo de disminuir la mortalidad infantil, teniendo como beneficiarias a las mujeres embarazadas a partir de la semana 12 del período de gestación, quienes deben cumplir con la totalidad de los controles prenatales requeridos por el sistema de salud público.

El acceso a todas las asignaciones universales por hijo está mediado por el registro en un sistema establecido por la Agencia Nacional de la Seguridad Social. En el caso de las ayudas universales por embarazo y aquellas por hijo, la condicionalidad del pago del monto estipulado queda sujeta al cumplimiento de los controles prenatales y los requisitos de escolaridad, control sanitario y plan de vacunación, respectivamente.

Debemos destacar un aspecto significativo señalado por los expertos que diferencia a la Ayuda Universal por Hijo de otras transferencias monetarias de América Latina: su incorporación al sistema de asignaciones familiares, como parte de un subsistema solidario no contributivo que extiende el derecho de la asignación por hijo a los trabajadores no registrados en la seguridad social (Lombardía y Rodrí-

13 Discurso de la Presidente Cristina Fernández de Kirchner en el lanzamiento del Programa Ingreso Social con Trabajo-Argentina Trabaja. Disponible en http://www.casarosada.gov.ar/ 
guez, 2015). De este modo, nos encontramos con la inscripción plena de los significados del dinero proveniente de las transferencias monetarias en el régimen de restitución y titularidad de derechos: en algunos de los documentos de las agencias de gobierno vinculados a la implementación de la ayuda universal por hijo (Ministerio de Trabajo, Empleo y Seguridad Social, 2012; Agencia Nacional de la Seguridad Social, 2012) encontramos claras asimilaciones a los enfoques regionales sobre las modificaciones en los sistemas de protección social impulsados por la CEPAL, ONU y la OIT.

Al igual que otras iniciativas de la región, la Ayuda Universal por Hijo consolida una narrativa de las transferencias monetarias bajo el paradigma de la titularidad de derechos económicos, sociales, políticos y culturales. Esta concepción permitirá considerar otras posibles entregas de dinero estatal bajo distintos formatos como, por ejemplo, las transferencias monetarias caracterizadas como "reparaciones históricas" (Luzzi, 2014).

Durante el transcurso de los años posteriores al surgimiento de la ayuda por hijo, se lanzan otra serie de medidas centradas en transferencias monetarias, entre las que podemos resaltar: el Programa de Respaldo a Estudiantes Argentinos (PROG.ES.AR) y las reformulaciones del Plan de Inclusión Previsional (2005-2015).

El Programa de Respaldo a Estudiantes Argentinos comprende una transferencia monetaria mensual dirigida a jóvenes de 18 a 24 años de edad que no trabajan, trabajan informalmente o perciben un salario mínimo vital y móvil, y quieren iniciar o completar sus estudios en cualquier nivel educativo.

El Plan de Inclusión Previsional (PIP) (Ley $\mathrm{N}^{\mathrm{o}} 25.995 / 2011$ ) surgió como una herramienta para que aquellas personas, que cumplían con la edad jubilatoria, pudieran hacerlo sin reunir el requisito específico de los 30 años de aportes o recurriendo al sistema de jubilación anticipada, aportando los últimos cinco años correspondientes a la edad mínima jubilatoria.

La iniciativa no solo favoreció el acceso a la jubilación a un número significativo de personas sino que impulsó la creación del Sistema Integrado Previsional Argentino (SIPA) que unificaba el sistema previsional, recuperando el Estado, a través de la figura de la Agencia Nacional de la Seguridad Social, los fondos del régimen de capitalización individual creados durante la década de 1990. Así, el Estado recobraba un área privatizada de la seguridad social, instaurando nuevamente el sistema de reparto, público y solidario (Arza, 2013). En este marco, la cobertura previsional en Argentina no solo alcanzó su máximo nivel histórico sino que representó la experiencia de mayor cobertura en Latinoamérica.

Este recorrido breve sobre el Plan de Inclusión Previsonal nos permite reflexionar en el alcance de las reformas recientes del sistema previsional y aludir a las moratorias de inclusión previsional como transferencias monetarias bajo distintos formatos: a) contributivo: para aquellos que lograron aportar la totalidad de los años requeridos; b)- semicontributivo: para aquellos que completaron sus años de aporte con las opciones previstas por las moratorias; y c) no contributivo: para el caso de aquellas personas que accedieron a la jubilación mínima sin registrar aportes de ninguna índole (Grushka, Casanova, Bertranou y Cetrangolo, 2012).

Nos interesa resaltar que dicho esquema, tripartito pero integrado, nos permite emparentar distintas transferencias monetarias estatales: la moratoria previsional da cuenta de la existencia de una transferencia monetaria significativa que, al igual que una medida trascendental como la Asignación Universal por Hijo, pone el acento en el dinero como puerta de entrada a garantizar efectivamente los derechos históricamente vulnerados.

\section{Las transferencias monetarias argentinas: intervención experta y formas plurales de accesibilidad}

En el recorrido que realizamos pudimos detenernos en el proceso de monetarización de las políticas sociales en Argentina y en una nueva interpretación de las formas de intervención en la pobreza. La introducción de las distintas medidas estatales nos permitió dar cuenta de una estrategia de intervención que tendió a generalizar las transferencias monetarias sobre distintos grupos sociales y aduciendo a distintas problemáticas asociadas a la pobreza como nueva cuestión social.

En poco más de una década del surgimiento de la primera iniciativa centrada en transferencias monetarias -Plan Jefes y Jefas de Hogar Desocupados- observamos un giro significativo en las narrativas sobre los programas: los conocimientos expertos, las premisas estatales 
y las agencias de gobierno circundaron desde el término de la crisis y la contención social, pasando por la necesidad de construir sistemas amplios/ integrales de protección social, para consagrar a las entregas de dinero estatal bajo el paradigma de los derechos.

También pudimos confirmar la relación entre los saberes expertos y las iniciativas en políticas sociales de transferencias monetarias que se consolidaron durante los últimos años en nuestro país. Observamos distintos debates expertos e institucionales estatales sobre las formas que debería de asumir la intervención monetaria sobre la pobreza. La emergencia de las políticas de entrega de dinero en nuestro país estuvo influenciada por una fuerte dependencia internacional, donde los expertos locales se constituyeron en los receptores/importadores de esos saberes y actuaron como reproductores de los mismos, generando discursos enmarcados en los antecedentes de nuestro país, y adaptando las recomendaciones a las tradiciones científicas y a la lectura nacional de los problemas sociales (Hornes y Maglioni, 2018).

A su vez, el desarrollo de este artículo nos permitió identificar distintas transformaciones propuestas por los expertos, sobre las lógicas de accesibilidad a las políticas de transferencias monetarias. La expansión de las transferencias monetarias en la Argentina da cuenta del rol de los expertos para construir una mirada legítima del dinero como elemento que reúne soluciones diversas sobre el fenómeno de la pobreza. En este sentido, y siguiendo las preocupaciones y los debates de los expertos, podemos afirmar que las entregas de dinero en el formato de transferencias monetarias lubricaron las formas de accesibilidad a las políticas sociales: a) el dinero de las transferencias monetarias garantiza el acceso a bienes básicos y favorece el consumo; b) tiene condiciones simples y realizables; c) resulta fácil de transferir y otorga transparencia institucional; d) es barato, en términos de gasto social; e) disuelve la intervención de mediadores sociales; y f) el dinero bajo el signo de los derechos permite pluralizar la accesibilidad en múltiples figuras de la asistencia (pobres, desocupados, niños y niñas, adolescentes, estudiantes, ancianos, grupos etarios heterogéneos y más allá de distinciones de género) (Barrientos, Hulme y Hanlon, 2008).

Cuando consultamos bibliografía sobre las políticas sociales, observamos que las barreras de accesibilidad se refieren a las siguientes dimensiones: la geográfica expresa el tiempo y la distancia que separa y dificulta la llegada de la población a los recursos; las barreras económicas se refieren a la imposibilidad de acceso en función de los recursos económicos disponibles; la dimensión administrativa alude a los aspectos burocráticos que se ponen en juego para acceder; las barrera cultural y simbólica remiten a los usos, costumbres, imaginarios sociales y representaciones de la población (Comes y Stolkiner, 2004; Comes, et al., 2006). Como sostuvimos a lo largo de este trabajo, la monetarización de las políticas sociales argentinas ha contribuido a flexibilizar o volver más permeables las barreras de la accesibilidad: el dinero y las cualidades atribuidas al mismo desde los saberes expertos en transferencias monetarias han jugado un rol fundamental en dicha operación, sumando innovaciones tecnológicas, administrativas y técnicas.

Sin embargo, debemos destacar que el gran saldo pendiente y poco problematizado por los expertos tras la monetización de las políticas sociales refiere a la calidad de los servicios públicos de salud y educación que acompañan a las transferencias monetarias. En este sentido, más allá de que en los últimos años se ha avanzado en leyes y políticas tendientes a ampliar la cobertura y la capacidad de protección de los servicios de salud y educación provistos por el Estado, el incremento de la demanda incentivado por las condicionalidades de las transferencias monetarias se enfrenta a una oferta sectorial que, en muchos casos, no ha logrado revertir las históricas dificultades de accesibilidad que caracteriza el vínculo de las familias pobres con las instituciones de salud y educación.

En este sentido, cabe señalar que la regularidad y estabilidad en la percepción de la transferencia monetaria ha introducido transformaciones en las prácticas que definen la cotidianidad de las familias destinatarias de estas prestaciones, posibilitando una ampliación de los márgenes de acción más allá de la reproducción básica y "nuevos" accesos, comportamientos, planificaciones y demandas que tensionan la oferta sectorial de servicios básicos, problematizando a la accesibilidad como proceso y empujando de modo necesario cambios en la oferta y su calidad.

En resumidas cuentas, a lo largo de este artículo hemos puesto a las transferencias monetarias en tensión con los procesos de la 
accesibilidad y los escenarios donde se llevan adelante dichos procesos; es decir: por un lado, en las secuencias institucionales y de demanda asistencial que han habilitado la accesibilidad ampliada a las transferencias monetarias y, por el otro, en las condiciones socio-históricas particulares (físicas, económicas, sociales y jurídico-normativas) en que se producen tales procesos.

El "giro a la derecha" de la región trae consigo un retroceso y deterioro en materia de protección social. En particular, en nuestro país, desde el inicio de la gestión presidencial de Mauricio Macri (2015) se pusieron en marcha un conjunto de políticas macroeconómicas que afectaron las fuentes de trabajo (formales e informales) y el poder adquisitivo de las familias como, también, cambios en la orientación de las políticas de protección y derechos sociales. En este sentido, podemos advertir el avance hacia un modelo de desarrollo en el que las políticas sociales se alejan del paradigma de la inclusión social con enfoque de derecho y se acercan a un enfoque subsidiario y residual similar al que conocimos durante la década de los '90 de hegemonía neoliberal. Consideramos que estos cambios tienen implicancias en materia de accesibilidad, replanteando el horizonte de las transferencias monetarias en el actual contexto al proponer nuevos "procesos" y "escenarios".

\section{Referencias bibliográficas}

Abramovich, V. y Pautassi, L. (2009). La revisión judicial de las políticas sociales. Estudio de casos. Buenos Aires: Editores del Puerto.

Agencia Nacional de la Seguridad Social. (2012). La Asignación Universal por Hijo para protección social en perspectiva. La política pública como restauradora de derechos. Buenos Aires: Observatorio de la Seguridad Social.

Arias, A. (2012). Pobreza y modelos de intervención. Aportes para la superación del modelo de asistencia y promoción. Buenos Aires: Espacio Editorial.

Arza, C. (2013). La política previsional y la prevención de la pobreza: elementos salientes de la trayectoria latinoamericana reciente. Revista Voces en el Fenix, 23. Monográfico Nosotros, los Pobres.

Barrientos, A., Hulme D. y Hanlon, J. (2008). Just give money to the Poor. The Development Revolution from the Global South. Manchester: Brooks World Poverty Institute.

Banco Mundial. (2009). Transferencias Monetarias Condicionadas: Reduciendo la pobreza actual y futura. Washington, DC: Banco Mundial.

Carballeda, A. (2014). La accesibilidad y las políticas de salud. En: Escenarios Sociales, Intervención y Acontecimiento. Buenos Aires: Ediciones digitales Margen.

CEPAL. (2006). La protección social de cara al futuro: acceso, financiamiento y solidaridad. Santiago de Chile: CEPAL/Naciones Unidas.

Clemente, A. (2010). Pobreza y territorio en áreas urbanas. En: A. Clemente (coord.), Necesidades sociales y programas alimentarios. Las redes de la pobreza. Buenos Aires: Espacio Editorial.

Clemente, A., Molina Derteano, P. y Roffler, E. (2014). Pobreza y acceso a las políticas sociales. El caso de los jóvenes en el conurbano bonaerense. Ciencias Sociales. Revista de la Facultad de Ciencias Sociales, 86, septiembre. Buenos Aires: Universidad de Buenos Aires.

Comes, Y. y Stolkiner, A. (2004). Si pudiera pagaría: Estudios sobre la accesibilidad simbólica de las mujeres usuarias pobres del AMBA a los servicios asistenciales estatales. Anuario de Investigaciones, XII. Buenos Aires: Facultad de Psicología-UBA/Secretaría de Investigaciones.

Comes Y. et al., (2006). El concepto de accesibilidad: la perspectiva relacional entre población y servicios. Anuario de Investigaciones, XIV. Buenos Aires: Facultad de Psicología-UBA/Secretaría de Investigaciones.

Elizalde, C. y Maglioni, C. (2014), Las redes como parte de la solución y como problema. En: A. Clemente (coord.), Territorios urbanos y pobreza persistente. Buenos Aires: Espacio Editorial.

Gruska, C., Casanova, L., Bertranou, F. y Cetrangolo, O. (2012). Encrucijadas en la seguridad social en Argentina: reformas, cobertura y desafios para el sistema de pensiones. Argentina: OIT.

Hornes, M. (2018). Políticas sociales y significados plurales del dinero: la producción social de las transferencias monetarias. [Tesis de Doctorado en Sociología. IDAES/ UNSAM].

Hornes, M. y Maglioni, C. (2018). Los Sistemas de Protección Social en América Latina y el Caribe. El rol de los saberes expertos en la expansión de los Programas de Transferencias Monetarias. Revista Debate Público. Reflexiones de Trabajo Social, 15/16 (inédito). 
Kessler, G. (2014). Controversias sobre la desigualdad. Argentina, 2003-2013. Buenos Aires: Fondo de Cultura Económica.

Luzzi, M. (2014). Pagar para reparar. Debates públicos y dilemas privados ante las políticas de reparación económica a las víctimas del terrorismo de Estado en Argentina. En: S. Gayol y G. Kessler (Ed.), Muerte, política y sociedad en la Argentina. Buenos Aires.

Lombardía, M.L. y Rodríguez, K. (junio 2015). La experiencia argentina en políticas de transferencias monetarias durante la última década. Cuaderno de trabajo, 7. Buenos Aires: Secretaría de Política Económica y Planificación del Desarrollo.

Lomelí, E. (2009). Conditional Cash Transfer programs: Achievements and illusions. Global Social Policy 9(2), 167-171.

Maglioni, C. (2018). Programas de transferencias condicionadas: protección social y producción de expectativas. De los debates regionales a la mirada de las familias destinatarias. [Tesis de Maestría en Intervención Social. FCS/UBA].

Ministerio de Trabajo, Empleo y Seguridad Social. (2012) ¿Piso o Sistema Integrado de Protección Social? Una mirada desde la experiencia argentina. Recuperado de: http://www.trabajo.gov.ar/downloads/seguridadSoc/pisoosistema.pdf

Repetto, F., Potenza, F. y Vilas, M. (2005). Plan Jefes y Jefas de hogar desocupados en Argentina (20022003). En: Un estudio de caso sobre la forma en que la política social se vincula a la emergencia política y socio-económica. Washington DC: Instituto Interamericano para el Desarrollo Social, BID.

Stolkiner, A. y Barcala, A. (2000). Reforma del sector salud y utilización de servicios de salud en familias NBI: estudio de caso. En: La Salud en Crisis. Un análisis desde la perspectiva de las Ciencias Sociales. Buenos Aires: Dunken.

Sugiyama, N. (2011). The diffusion of Conditional Cash Transfers programs y Latin America. Global Social Policy, 11(2-3), $250-278$.

Teichman, J. (2007). Multilateral lending institutions and transnational policy networks in Mexico and Chile. Global Governance 13(4), 557-573.

Uña, G., Lupica, K. y Strazza, L. (2009). Think tanks y pobreza en América Latina: el rol de los pensadores en el mercado de las políticas sociales en Argentina, Chile y México. Revista del CLAD Reforma y Democracia, 44, 225-266. Junio.

Vommaro, G y Morresi, S. (2012). Saber lo que se hace. Política y expertise en Argentina. Buenos Aires: Prometeo-UNGS. 